CAHIERS DE

NARRATOLOGIE

\section{Cahiers de Narratologie}

Analyse et théorie narratives

31 Bis | 2017

Espace du récit, récit de l'espace en contexte germanique

\title{
Le tapis d'orient en Allemagne et en Autriche à la fin du XIXe siècle : défi spatial, défi narratif
}

\section{Céline Trautmann-Waller}

\section{(2) OpenEdition}

\section{Journals}

Electronic version

URL: http://journals.openedition.org/narratologie/7751

DOI: $10.4000 /$ narratologie. 7751

ISSN: 1765-307X

Publisher

LIRCES

Electronic reference

Céline Trautmann-Waller, « Le tapis d'orient en Allemagne et en Autriche à la fin du XIXe siècle : défi spatial, défi narratif

», Cahiers de Narratologie [Online], 31 Bis | 2017, Online since 26 June 2017, connection on 04 May 2019. URL : http://journals.openedition.org/narratologie/7751 ; DOI : 10.4000/narratologie.7751

This text was automatically generated on 4 May 2019.

Article L.111-1 du Code de la propriété intellectuelle. 


\title{
Le tapis d'orient en Allemagne et en Autriche à la fin du XIXe siècle : défi spatial, défi narratif
}

\author{
Céline Trautmann-Waller
}

1 On constate durant le dernier tiers du XIXe siècle un intérêt inédit pour le tapis d'orient de la part des historiens d'art, collectionneurs et consommateurs, en Europe et peut-être tout particulièrement en Allemagne et en Autriche, où a lieu en 1891 la première exposition exclusivement consacrée aux tapis d'orient. Autrefois objet de luxe utilisé à des fins de représentation dans le contexte royal et aristocratique, le tapis d'orient devient petit à petit et parallèlement un élément (standard) de l'intérieur bourgeois et une pièce de musée élevée au rang d'objet d'étude de l'histoire de l'art, tant il est vrai que le commerce du tapis n'inclut pas seulement l'approvisionnement en tapis mais aussi l'approvisionnement en informations à leur sujet ${ }^{1}$. Résultant à la fois d'une transformation du goût, de nécessités économiques et d'une situation géopolitique, ce que l'on pourrait appeler l'« ère bourgeoise » du tapis d'orient met en effet en relation, au moyen de divers types de récit (récit historique, récit de voyage, récit de soi), des espaces « orientaux» concrets et un orient mythique, l'espace du musée ou de l'intérieur et l'espace ornemental du tapis lui-même.

2 Les premières publications scientifiques sur les tapis d'orient paraissent à partir des années 1870. Elles sont le fait d'historiens de l'art allemands et autrichiens comme Julius Lessing, Wilhelm Bode, ou Alois Riegl. Dans leurs études, les étonnantes pérégrinations des tapis de pays en pays, au gré des échanges commerciaux, des relations diplomatiques ou des guerres, prolongent pour ainsi dire le nomadisme auquel le tapis - habitat portatif - reste associé. Mais plus encore, ce sont les voyages des techniques et des motifs, ces derniers changeant aussi de support et se retrouvant jusque dans les tableaux de la Renaissance européenne, qui constituent un défi pour le récit historique tendu entre appropriation et défamiliarisation.

3 Parallèlement, l'intégration des tapis d'orient à différents espaces, comme le musée ou l'intérieur, pose un certain nombre de problèmes à la fois pratiques et symboliques. 
L'analyse des «stratégies " spatiales développées pour y répondre, montre combien le tapis d'orient, bien qu'étant un élément familier de l'iconographie européenne, reste associé à des modes de vie particuliers et, loin de seulement décorer les espaces auxquels il est intégré, introduit dans ceux-ci comme un regard extérieur. Tant il est vrai que le système ornemental dont le tapis d'orient est issu correspond lui-même selon divers travaux de l'époque à une certaine perception de l'espace. C'est aussi la représentation de l'espace, propre à l'art européen depuis l' « invention » de la perspective, que le tapis met à l'épreuve puisque, tout comme d'autres arts « extra-européens » découverts en cette fin du XIXe siècle, il paraît contraster avec une figuration occidentale de l'espace. D'autre part le tapis apparaît comme incarnation d'un art du motif, à la fois exotique et particulièrement apte à l'appropriation en raison de son abstraction. Si l'on tient compte du fait que l'abstraction et la planéité, ouvrant toutes deux sur la matérialité et le rythme, vont devenir un des éléments de la modernité picturale, on peut se demander dans quelle mesure le tapis d'orient participe aussi à sa manière à un autre (grand) récit, celui du modernisme, ici sous l'aspect de la modernité picturale.

\section{Le tapis d'orient, le récit historique et l'espace des relations entre orient et Europe}

4 L'essor et la valorisation des arts décoratifs comme domaine artistique à part entière fait que bien des collections princières sont "recyclées" au XIXe siècle en collections muséales. C'est une des origines principales des tapis dans les collections des arts décoratifs, par exemple à Vienne, où cette dimension est particulièrement nette ${ }^{2}$, alors que d'autres collections de tapis sont issues des activités de collectionneurs privés ou ont été constituées plus tard par des conservateurs dans un but scientifique (les deux pouvant aussi aller de pair). Cette mutation est liée à l'émergence d'une approche "scientifique " $\mathrm{du}$ tapis d'orient qui implique des recherches sur les provenances, les techniques, les motifs. Tandis qu'ils étaient souvent précédemment qualifiés de manière générique de "persans» ou de "turcs " ${ }^{3}$, ce qui ne désignait pas un lieu de fabrication concret mais plutôt un style, les tapis orientaux donnent lieu désormais à une véritable géographie ${ }^{4}$ et à une cartographie, comme le montrent les premières publications et, de manière paradigmatique, la classification des tapis et la disposition des salles lors de la première grande exposition internationale consacrée exclusivement aux tapis d'orient organisée à Vienne en 1891, sur laquelle nous reviendrons.

Avant même cette exposition, les publications de Julius Lessing avaient initié la " découverte du tapis ${ }^{5}$ par les historiens d'art. Grand admirateur de Gottfried Semper qui, dans son livre sur le style dans les arts tectoniques etc., avait attribué au textile une place primordiale dans l'histoire de l'ornementation et des arts décoratifs ${ }^{6}$, Julius Lessing s'était qualifié comme expert de ces derniers en écrivant des comptes rendus des expositions universelles pour la presse, avant de devenir directeur du Musée des arts décoratifs (Kunstgewerbemuseum) de Berlin. Il raconte dans un récit autobiographique comment, fasciné par les tapis d'orient représentés dans divers tableaux de la Renaissance européenne, il eut l'idée de les reproduire et de les classifier, avant de s'intéresser à l'objet tapis en lui-même ${ }^{7}$. Son ouvrage de 1877, Die orientalischen Teppichmuster des XV. und XVI. Jahrhunderts ${ }^{8}$, traduit en anglais et en français dès $1879^{9}$, est généralement considéré comme le premier ouvrage scientifique (au sens de l'histoire de l'art) consacré aux tapis d'orient. Le récit complexe de sa quête des sources et de l'origine 
des tapis et surtout la reconstitution du voyage des motifs, de pays en pays, met en relation espaces «occidentaux » et " orientaux », et esquisse une histoire de l'art globale et hybride.

6 L'ouvrage est conçu autour d'un ensemble de trente planches comportant un ou plusieurs dessins de motifs de tapis issus de tableaux, principalement flamands et italiens, des XVe et XVIe siècles, exécutés par Lessing lui-même. Le texte consiste en une introduction générale, une présentation historique ainsi que des explications et des commentaires des planches. Dans la première partie de l'introduction Lessing expose ses sources et sa méthode. Il y raconte comment, de musée en église et d'église en collection privée, il a effectué un "travail de collecte et de reconstitution " (zusammenstellende Arbeit) ${ }^{10}$ inédit. Si chez certains peintres, comme Holbein, il a pu trouver des représentations très exactes mais partielles des motifs, chez d'autres, comme les Vénitiens, les représentations sont moins précises mais offrent la totalité du motif. En associant les deux, il a cherché à atteindre son but, celui d'une "redécouverte", ou «reconquête", des motifs (Wiedergewinnung der Muster) ${ }^{11}$. Parfois un même motif est représenté de manière différente, comme par exemple sur la planche 23, où Lessing compare la représentation d'un motif dans deux tableaux différents, l'un proposant une reproduction originale du motif, l'autre une "traduction de ces formes [orientales] dans la langue formelle européenne ». Ce cas pose selon Lessing la question de savoir qui a opéré cette traduction et la question plus générale des «remaniements européens» (die europäischen Umgestaltungen) à l'œuvre dans les représentations de tapis d'orient ${ }^{12}$.

7 Lessing passe ensuite à la technique et indique que les tapis représentés dans les tableaux sont pour l'essentiel des tapis destinés à être posés sur le sol (Fussteppiche) - un type de tapis inventé en orient selon lui - même si en Europe ils sont aussi utilisés pour recouvrir des tables ou d'autres meubles ${ }^{13}$. Par ailleurs il s'agit pour tous, excepté pour trois d'entre eux, de tapis noués, selon une technique orientale ancestrale qui n'est pas attestée en Europe avant le XIXe siècle, où elle est apprise de manière systématique en Asie puis introduite en Europe $^{14}$. Lessing évoque une mission organisée par le Ministère du commerce prussien à l'instigation de la chambre de commerce de Görlitz en 1853 : deux techniciens, les fabricants de tapis Kühn de Kottbus et Böhm de Schöneberg en Silésie, ont été envoyés en Asie mineure (à Ghiordès (Gördes), Kula et Uschak (Uşak), principaux lieux de fabrication des tapis dits de Smyrne, tous situés à quelques jours de voyage de Smyrne) pour y étudier le travail du tapis. Lessing cite les comptes rendus de cette mission indiquant que les voyageurs ont retrouvé partout les mêmes modes de fabrication et les mêmes métiers à tisser et que partout seules des femmes travaillent sur ceux-ci. Pour ce qui est de l'ornementation, les rapports indiquent que les tapis sont réalisés à Ghiordès par exemple dans quatre ou cinq motifs seulement, et ce d'après des fragments de tapis transmis dans les familles par héritage, ou bien sur commande depuis Smyrne d'après des motifs que les femmes arméniennes y dessinent pour des fichus turcs. Ces derniers temps les commandes seraient venues aussi directement d'agents européens. Ce voyage d'étude a rendu possible selon Lessing une connaissance exhaustive de la fabrication des tapis et son introduction en Silésie, puis en Saxe, en Palatinat, en Bohême, à Vienne, en Belgique. Lessing souligne toutefois que toutes les tentatives pour perfectionner les métiers à tisser européens pour permettre la réalisation de tapis semblables aux tapis d'orient ont échoué : «On travaille de nouveau partout à la main comme dans le désert asiatique. $»^{15}$. 
8 Pour ce qui est des motifs, Lessing souligne combien le principe du nouage et les contraintes techniques qui y sont liées ont influencé les motifs, par opposition aux techniques européennes qui incitent selon lui à l'arbitraire dans le choix des motifs. Suivant en cela Semper, cité ici, Lessing considère que, tout comme les mosaïques dans l'Antiquité, dont les figures étaient soumises à certaines contraintes géométriques, les motifs propres aux tapis noués, et notamment les motifs géométriques, conviennent particulièrement pour la décoration des sols, parce qu'ils n'attirent pas trop le regard. Dans le cas de tapisseries murales comme les Gobelins, les conditions techniques et stylistiques sont autres, puisqu'il s'agit de réaliser pour ainsi dire des tableaux. Le tissage, organisé en principe autour de la reprise d'un même motif, ne suffit plus alors, et il faut passer à la haute-lisse, que Lessing définit comme un intermédiaire entre tissage et broderie.

Dans le panorama historique qui suit, Lessing cite différentes sources concernant l'usage des tapis durant l'antiquité et évoque notamment une reproduction de tapis en albâtre. Selon Lessing ces sources montrent que les tapis asiatiques étaient un objet de luxe largement répandu chez les Grecs et les Romains, mais elles donnent très peu d'informations sur l'origine de ceux-ci, encore moins sur les techniques et les motifs ${ }^{16}$. Le moyen âge s'est fourni en tissages de luxe et soieries, dont il faisait un usage massif, presque exclusivement en orient, si on y inclut la Sicile et l'Espagne qui, à l'époque, étaient orientales selon Lessing. Il n'existe pas en revanche d'indications sur une utilisation des tapis pour décorer les sols. Les "tapis sarrasinois » que l'on trouve dans différentes sources et qui étaient fabriqués à Paris, n'étaient pas selon Lessing des tapis noués destinés à décorer le sol, mais des tapisseries murales avec des éléments figuratifs. Dès le XIIIe siècle l'importation de Gobelins devint si massive en Europe que tous les tapis de ce genre furent qualifiés de "sarrasinois » en référence à cette origine orientale, et que même au XVe siècle, lorsque la fabrication européenne se fut largement autonomisée, le souvenir de cette origine asiatique n'avait pas disparu. La véritable introduction du tapis à peluche oriental dans l'industrie européenne ne lui paraît attestée qu'après 1600 et, même à cette date, seulement de manière limitée.

10 Les sources textuelles ne donnant pas d'indication sur les techniques et les motifs, Lessing s'est tourné vers les reproductions visuelles et quelques spécimens originaux pouvant servir de sources. Il existe, certes, des représentations de tapis d'orient dans des manuscrits du moyen âge mais elles sont difficilement reconnaissables selon lui. Plus riches sont les fresques de bâtiments italiens du moyen âge. Dans la basilique de San Miniato, la marqueterie en marbre du sol est inspirée des motifs de tapis d'orient ${ }^{17}$. Mais ce sont avant tout les tableaux de la Renaissance qui offrent des sources de grande valeur pour la connaissance des tapis d'orient des XVe et XVIe siècles, Lessing soulignant tout particulièrement le rôle de Venise dans ce domaine.

11 Comme l'indique de manière symptomatique l'index des noms de lieux à la fin de cet ouvrage, où l'on retrouve Babylone à côté de Bamberg, Constantinople à côté de Coraxer, Le Caire entre Innsbruck et Kassel, Niniveh ente Naples et Nuremberg, l'histoire des tapis d'orient apparaît comme une des premières formes d'une histoire globale de l'art. La question de la circulation des motifs et des techniques, et notamment celle des imitations européennes, de la Savonnerie parisienne jusqu'aux fabricants du XIXe siècle, révèle combien elle est aussi une histoire hybride. Julius Lessing a du mal néanmoins à proposer un récit qui permettrait d'unifier tous ces phénomènes, d'expliquer les liens, de comprendre les réseaux qui les ont rendus possibles. Le terme d' «histoire de 
l'évolution » (Entwicklungsgeschichte) revient sous sa plume, mais les relations retracées restent partielles.

Dans sa deuxième publication concernant les tapis d'orient, un volume de la série des «cahiers de modèles» (Vorbilder-Hefte) consacré à cette question et paru en $1891^{18}$, Lessing raconte que, convaincu au départ qu'il fallait se contenter des tapis représentés dans les tableaux et que ceux-ci suffisaient comme sources, il a compris depuis que ce n'était pas le cas. Il retrace la constitution de la collection de tapis d'orient au Musée des arts décoratifs de Berlin: "Cette collection a été entamée par Monsieur le Directeur Grunow en 1871, qui pour commencer fit venir à Berlin une grande quantité de tapis d'orient anciens et modernes par un marchand de tapis viennois et les exposa au Musée des arts décoratifs; nous devons des acquisitions importantes également à l'aide aimable de Monsieur le Conseiller Dr. Bode. La collection comprend désormais, après rejet de nombreux doublons et de pièces de peu de valeur, 225 tapis et fragments, presque tous de facture orientale ancienne. La plupart ont été trouvés dans des églises allemandes, plus encore italiennes, où depuis leur transfert depuis l'orient, elles ont été mises de côté après avoir commencé à se détériorer, et ont ainsi été conservées. Une partie provient aussi directement d'Espagne ou de l'orient. $»^{19}$

Cet ouvrage est organisé de manière à permettre au lecteur - on devrait dire à l'utilisateur - d'utiliser les planches séparément comme modèles, puisque les tapis euxmêmes, très fragiles, ne peuvent pas toujours être prêtés. Le livre devient une collection mobile. L'image prend le pas sur le texte - Lessing commente longuement l'utilisation de la photographie -, la collection sur le récit, mais ces glissements posent aussi la question de la narrativité des collections elles-mêmes.

\section{Le tapis d'orient et la narration muséale : l'exposition de 1891}

Les tapis d'orient étaient déjà présents dans les expositions universelles organisées depuis le milieu du siècle dans différentes villes européennes (Londres 1851, Paris 1855, Londres 1862, Paris 1867, Vienne 1873, Paris 1878, Melbourne 1880, Barcelone 1889) et les comptes rendus soulignent dès cette époque leur grande qualité décorative. L'exposition de tapis organisée au Musée du commerce à Vienne en 1891 marque néanmoins un tournant important.

L'exposition universelle de 1873 à Vienne avait donné naissance à un Cercle oriental qui, lui-même, fonda un Musée oriental (installé dans un espace au sein du bâtiment de la Bourse), alimenté en partie avec des acquisitions faites à l'occasion de l'exposition ${ }^{20}$. Le Musée oriental fut dirigé depuis le début par Arthur von Scala ${ }^{21}$, qui avait été chargé précédemment par le Ministère autrichien de l'économie et du commerce d'un rapport sur l'industrie textile lors de l'exposition universelle de 1867 et avait participé comme spécialiste du textile à une expédition autrichienne en Chine, au Siam et au Japon, organisée entre 1868 et 1871 pour permettre l'ouverture de relations commerciales avec ces pays. Au Musée oriental, von Sala organisa en 1884 une exposition sur les céramiques orientales. L'exposition de 1891, quant à elle, eut lieu après la transformation du Musée oriental en Musée du commerce. De la disposition des salles à la préparation du catalogue et de l'ouvrage reproduisant en noir et blanc et en couleur une bonne partie des tapis exposés, on peut dire que l'expositions expérimenta un nouveau traitement 
muséographique et iconographique du tapis et marqua un moment essentiel de son entrée dans l'histoire de l'art, donc dans l'espace académique et dans le musée. Elle donna l'occasion de réfléchir à la manière dont on pouvait rendre justice aux tapis d'orient dans l'espace d'un musée. Cette question entretient un rapport intime avec les recherches des historiens d'art et leurs récits puisque, comme il a encore été souligné récemment, à travers ses collections et leurs modes d'exposition le musée "performe des histoires d'art » (stories of art) et qu'« un objectif principal pour les musées est toujours de narrer sur l' « art » (...) ( (A chief aim for museums is still to narrate about « art $»^{22}$ ).

16 A ce jour aucune photographie de l'exposition de 1891 n'a pu être retrouvée, mais le catalogue comprend un plan de cette dernière, indiquant la disposition des salles et la répartition des tapis - plus de 400 pièces - dans celles-ci. Il fait apparaître un mélange de regroupements thématiques (tapis de prière, intérieur, imitations de meubles anciens) et géographique (Perse, Asie centrale, Bosnie, Smyrne et Inde, Chine, Turkestan et Japon, Tunisie et Maroc). Notons également que Arthur von Scala indique dans la préface du catalogue qu'on fit appel pour l'installation des tapis à un artiste, en l'occurrence le jeune peintre Hugo Charlemont ${ }^{23}$. Les publications liées à l'exposition combinent, dans les textes, le souci de la compréhension et de l'acquisition des techniques orientales, la classification de ceux-ci selon l'origine géographique, les époques et les styles, la mise en valeur de la maitrise des couleurs et des motifs, le caractère transculturel de ces derniers. Les illustrations des deux ouvrages jouent à cet égard un rôle stratégique. Arthur von Scala rappelle dans la préface du catalogue les mérites de l'Ecole impériale et royale et Laboratoire d'Essais pour la photographie et les procédés de reproduction (k.k. Versuchsanstalt für Photographie und Reproductionsverfahren) en indiquant qu'ils apparaitront plus nettement encore dans l'ouvrage ultérieur. Ce regard photographique sur les tapis (parallèlement en noir et blanc et en couleur dans le deuxième ouvrage) contribua grandement à leur transformation en œuvres d'arts. Il renforçait encore la planimétrie et l'effet de surface, sur lesquels nos reviendrons.

17 Un grand nombre de personnes furent associées de près ou de loin à cette vaste exposition, comme l'indiquent les remerciements dans le catalogue. Parmi les exposants ${ }^{24}$ , figurent des musées, des maisons royales, des diplomates, des collectionneurs, des marchands, tandis que la liste des assistant ${ }^{25}$ comprend des connaisseurs de l'orient, des historiens d'art, des artistes. L'un des exposants était le directeur de musée berlinois Wilhelm von Bode qui avait commencé à s'intéresser aux tapis d'orient lors de son premier voyage en Italie en 1871 et écrivait dans ses mémoires que « lors de ces premiers voyages en Italie je trouvais également ici ou là dans des églises et des ateliers d'artistes des tapis d'orient, dont la concordance avec les tapis apparaissant sur des tableaux me frappa $»^{26}$. Dès cette époque, mais surtout durant les années 1880 , Bode acheta des tapis, pour lui-même mais aussi pour ses amis artistes et collectionneurs ou pour des musées. Bode mentionne également l'exposition viennoise dans ses mémoires, considérant qu'elle «était très hétérogène et sans goût dans la disposition. De la modeste marchandise moderne était accrochée à côté de merveilleux tapis anciens, de manière aussi désordonnée que dans un vulgaire bazar turc, mais par le nombre de pièces anciennes de grande valeur et l'excellente publication parue peu de temps après, l'exposition a eu malgré tout un effet très stimulant sur l'étude de l'art islamique $»^{27}$.

18 Il est à noter que l'année suivant l'exposition viennoise, Bode, exemplifiant l'effet stimulant de cette dernière, publia ses deux premiers articles sur les tapis d'orient : l'un dans la revue des collections d'art prussiennes à Berlin ${ }^{28}$, l'autre précisément dans le livre 
issu de l'exposition viennoise ${ }^{29}$. Il s'agissait pour cette dernière d'une contribution concernant un tapis faisant partie de sa collection particulière, prêté comme d'autres pour l'exposition. Il republiera ultérieurement ces deux textes sous la forme d'un manuel sur les anciens tapis noués du Proche-Orient, écrivant ainsi sa propre histoire du tapis ${ }^{30}$. A Berlin, plusieurs musées se trouvaient en concurrence pour héberger des tapis d'orient: le musée des arts décoratifs, dirigé par Lessing, le musée ethnologique et les musées dont Bode fut successivement le directeur. Notons que c'est en 1892, donc un après l'exposition viennoise, que Bode commença lui-même à expérimenter des stratégies d'exposition pour les tapis d'orient. Inspiré par la manière dont les tapis d'orient avaient été recyclés comme éléments de décoration dans des églises en Italie et par l'impression que lui fit la représentation de Madonnes devant des tapis dans la peinture italienne et hollandaise, il exposa dans une salle du Altes Museum une Madonne de Benedetto da Maiano devant un tapis persan acquis peu de temps auparavant à Venise. Selon Bode, qui allait ici beaucoup plus loin que les historiens d'art de son époque, il existait une dépendance étroite entre les couleurs de la peinture vénitienne et flamande et les tapis reproduits dans les tableaux. La vivacité des couleurs de la peinture vénitienne du XVe siècle, surtout, s'était développée selon lui dans la rencontre avec le tapis d'orient, les couleurs ayant migré des tapis représentés dans les tableaux, vers les tableaux eux-mêmes ${ }^{31}$.

Lorsque Bode développa son projet d'une section d'art islamique au sein du FriedrichWilhelm-Museum (aujourd'hui Bode-Museum), il réclama pour ce dernier une grande partie des tapis d'orient présents dans les collections berlinoises. Lessing, qui souhaitait conserver l'importante collection de tapis d'orient constituée par ses soins au Musée des arts décoratifs, s'y opposa en argumentant de la façon suivante: « Les tapis ne sont pas pour nous des pièces d'exposition étrangères appartenant à l'Islam, mais ces tapis, qui ont été préservés jusqu'à nos jours presque sans exception dans des églises européennes, constituent des composantes essentielles de la culture européenne et ont exercé une si profonde influence sur l'art européen qu'ils sont bien plus importants pour la culture générale et l'enseignement en rapport avec l'art local, que dans leur rapport avec l'Islam $»^{32}$.

20 La question de l'appropriation européenne des tapis d'orient, liée ici au récit du sauvetage puis de la redécouverte des tapis dans les églises, se pose également dans le cas de l'intérieur.

\section{Le tapis et l'espace de l'intérieur bourgeois : orientalisation de l'intérieur et récit de soi}

21 On peut noter tout d'abord, qu'en cette fin de XIXe siècle les échanges entre musée et intérieur sont nombreux: les musées exposent des intérieurs prélevés ou reconstitués (les period rooms); les intérieurs, marqués par l'historisme, intègrent des objets de différentes époques et de différentes régions du monde et ressemblent à des musées en miniature. Dans les deux cas, on peut constater une mode orientale ou orientalisante, où le tapis joue un rôle central. Dans le cas de l'Autriche notamment, cette mode a pu être considérée comme faisant partie d'un "colonialisme visuel, plus exactement (d')un colonialisme de l'imaginaire » que la monarchie austro-hongroise, quasiment dépourvue de possessions coloniales, aurait pour cette même raison pratiqué de manière intensive ${ }^{33}$. On retrouve cette mode orientalisante notamment à la cour, puisque dans l'aménagement de la Hofburg, les tapis à motifs floraux de la manufacture de tapis de Linz, qui avait cessé 
son activité en $1850^{34}$, sont à l'époque remplacés par des tapis d'orient fabriqués en grande partie par la maison Haas ${ }^{35}$. Le prince-héritier Rudolf pour sa part aménagea, après son voyage en Orient de 1881 dont il publia le récit en $1885^{36}$, au sein de la Hofburg une " chambre turque $~^{37}$, dans laquelle de nombreux tapis d'orient recouvraient le sol et les divans. Au Musée des arts décoratifs (Museum für Kunst und Industrie) de Vienne la «Chambre arabe » (Arabisches Zimmer), inaugurée en 1883, célèbre pour l'inspiration qui trouvera Le Corbusier lors de son séjour à Vienne en 1907/08, comportait un tapis de Smyrne sur le sol de la pièce principale et des tapis du Daghestan sur les banquettes de la niche ${ }^{38}$.

Grâce à une promotion efficace, notamment dans le cadre des expositions universelles, le tapis d'orient (qu'il s'agisse de tapis anciens ou de tapis de fabrication récente, de tapis effectivement fabriqués en orient ou de tapis fabriqués en Europe selon différents procédés d' «imitation») devient également un élément de l'intérieur bourgeois ${ }^{39}$. Comme le note Alois Riegl, «dans les cercles les plus larges, non pas seulement de la population plus aisée, c'est désormais pour ainsi dire un point d'honneur de posséder au moins un authentique tapis d'orient $»^{40}$. Associé aux débats de l'époque sur les origines de l'architecture, le tapis tend même à devenir une icône de l'habitat/ion. Pour certains historiens de l'art, les tapis tissés constituèrent effectivement une des premières manières de créer un espace habitable, liée selon eux au nomadisme, que ce soit dans la péninsule arabe ou en Asie centrale. Carl Bötticher, professeur à l'Académie d'architecture (Bauakademie) de Berlin, analysant le temple grec comme une «tente idéale », considère que les tapis tissés ont été créés dès l'origine pour être des surfaces produisant de l'espace, que ce soit en couvrant, en formant une clôture, ou sous forme de tapis de sol, et note que les Arabes traitent jusqu'à aujourd'hui « tout le bâtiment comme une tente tendue de tapis $»^{41}$. Gottfried Semper voit lui aussi des analogies entre les tapis et les éléments de l'architecture, comme le mur, le plafond ou le sol, mais il les envisage de manière plus génétique que symbolique. ${ }^{42}$

Dans les comptes rendus de l'exposition universelle de 1873 rédigés par Jakob von Falke, futur directeur du Musée d'art et d'industrie de Vienne, on peut suivre l'histoire de la percée du tapis d'orient dans les intérieurs européens, dans un récit où l'orient en vient à être personnifié : "Lorsqu'en 1851 à l'exposition universelle de Londres l'orient se montra pour la première fois dans une certaine clarté, il constitua une apparition tout à fait nouvelle et bizarre. Le public ne le comprit pas et le contempla telle une chose tout à fait étrangère comme avec des yeux d'enfant étonnés. Quelques artistes et amis de l'art seulement devinaient à l'époque qu'il était le seul en réalité dans certains secteurs de l'artisanat d'art à posséder les bons principes, le seul qui avait de la couleur et pouvait nous apprendre la couleur, et non pas le goût français tant loué. Entretemps l'orient était devenu une apparition intéressante, comme un étranger célèbre, et l'intérêt crut d'exposition en exposition $»^{43}$.

Le regard historique sur les tapis d'orient dans la peinture européenne permet de poser la question de la compatibilité entre ornement oriental et styles européens et de l'harmonie des couleurs qui acquièrent une nouvelle actualité : « Nous trouvons très fréquemment sur les tableaux des anciennes Ecoles, de l'Ecole italienne tout comme de l'Ecole allemande ou néerlandaise, depuis le XVe jusqu'au XVIIe siècle, l'utilisation de tapis d'orient. Nous pouvons certainement en conclure qu'ils se trouvaient aussi fréquemment dans les appartements, et nous le savons de plus pour ce qui concerne Venise grâce à différents récits. Sur les tableaux ces tissus orientaux et leurs motifs très particuliers, 
généralement géométriques, mais travaillés de manière très désordonnée, s'accordent très bien avec les différents styles de l'appartement, qu'il s'agisse du style gothique ou de la Renaissance ou de la manière plus tardive que nous représentent les peintres de tableaux de genre hollandais, et notre rigorisme n'est pas heurté par leur utilisation. L'artiste les aime en raison de leur caractère coloré et les utilise pour l'effet pittoresque. Nous n'avons pas besoin de faire autrement dans l'appartement. Sans véritable style, que précisément nous n'avons pas, l'harmonie des couleurs est le premier point de vue que nous devons observer dans l'aménagement de notre appartement et dans ce domaine aucun matériau supérieur au tapis d'orient ne s'offre à nous. Soyons donc sans rancune à l'égard de ce trait de notre époque qui ramène aujourd'hui cette belle décoration dans notre appartement. $»^{44}$.

Les manuels de décoration indiquent eux-aussi combien l'intégration du tapis d'orient, si d'un côté elle paraît aller de soi, n'en pose pas moins un certain nombre de questions. On peut citer à titre d'exemple de nouveau Jakob von Falke et l'ouvrage L'Art dans la maison (Die Kunst im Hause, 1871), qui est à la fois une histoire de la décoration intérieure de l'antiquité jusqu'à son époque et un manuel de décoration ${ }^{45}$. Le succès de cet ouvrage, qui reprenait et développait des conférences tenues au Musée d'art et d'industrie de Vienne, est attesté par les cinq éditions qui virent le jour entre 1871 et 1883 dans différents formats, et par la parution, en 1879 aux Etats-Unis, d'une édition anglaise avec des illustrations en couleur. A différents endroits, Falke tente de répondre à la question de savoir comment on peut utiliser à bon escient le tapis d'orient dans la décoration intérieure de son époque. Certes, il part du principe que la compatibilité que l'on constate dans les anciens tableaux fonctionne également dans les appartements et qu'on peut donc suivre l'exemple des peintres ${ }^{46}$, mais ses développements montrent bien que l'intégration du tapis n'est pas sans poser de nombreuses questions et demande des aménagements ${ }^{47}$. La première question est sans doute de savoir dans quelle pièce il convient de le placer : dans le boudoir, le fumoir ou le salon? Ensuite, celle de savoir s'il convient de le poser à terre, ou d'en recouvrir sofas, tables etc, sachant que les deux usages allaient de pair à cette époque.

Au-delà d'un nomadisme des origines, de nature assez floue et mythique, différentes pratiques culturelles, concrètement expérimentés lors de voyages ou bien associés à un imaginaire de l'orient, s'incarnent dans le décor oriental dont le tapis est un élément central, tout en tendant à s'autonomiser. Ce qu'on peut analyser comme une orientalisation de l'espace privé constitue une invitation à des postures nouvelles, à une nonchalance opposée au rythme effréné de la vie moderne, mais aussi à la rêverie, comme on peut le voir par exemple lorsque Le Corbusier commente la chambre arabe du Musée d'art et d'industrie de Vienne : «les divans dans les niches: c'est un paradis pour toute personne qui sait rêver $»^{48}$. On peut insister sur la standardisation des goûts et la production de masse, c'est-à-dire d'une certaine manière sur la banalisation du tapis d'orient, mais comme le montrent ces exemples, l'intérieur est pourtant, tout comme le musée, un espace qui prétend raconter une histoire, offrir une performance du sujet, ainsi que le révèlent par ailleurs également les nombreuses descriptions d'intérieurs dans la littérature. Le fait que le divan sur lequel Freud faisait allonger ses «patients » ait été recouvert d'un tapis d'orient, associe également de manière symptomatique le tapis d'orient et le récit de soi $^{49}$. 


\section{L'espace ornemental du tapis d'orient : la planéité, l'histoire immanente des motifs et l'émergence de la modernité picturale} fabrication (tapis noués, tapis tissés), aux couleurs et aux matières (soie, laine), mais il faut noter surtout que le tapis d'orient joue un rôle important pour les études sur l'ornementation orientale et que les questions liées à la spatialité y occupent une place centrale.

La première chose qui frappe les collectionneurs de tapis orientaux, c'est que dans leur grande majorité, ils ne comportent pas de scènes figuratives et donc d'une certaine manière ne "racontent " pas, par opposition notamment aux tapisseries européennes. C'est peut-être d'autant plus étonnant que l'orient est généralement associé à l'époque en Europe au conte, forme paradigmatique du récit. Ca l'est deuxièmement aussi parce que, dans les langues européennes, le récit, et plus généralement l'activité littéraire, est associé à toute une série de métaphores textiles: le fil ou la trame du récit, le texte comme tissu (texture, textilité ${ }^{50}$ ). Si les tapis ne « racontent » pas, ils paraissent étrangers également aux traditions picturales européennes, centrées depuis la Renaissance sur la perspective, puisque leur décor est généralement bi-dimensionel, ou planimétrique. C'est d'ailleurs cette planéité qui a peut-être suscité par contraste l'idée du tapis volant, icône du conte oriental en Europe. Ce contraste peut paraître étonnant dans la mesure où la « découverte » de la perspective peut être mise en rapport, comme l'a fait Hans Belting, avec un mathématicien arabe du XIe siècle, Alhazen, qui créa certes les conditions pour la peinture en perspective occidentale, mais sans que cela n'ait eu de conséquence pour les traditions figuratives orientales, marquées - toujours selon Belting - par l'interdit de la représentation. Il fallut attendre la Renaissance européenne, pour que les acquis de la géométrie arabe soient redécouverts et finalement traduits en images dans la peinture européenne ${ }^{51}$. A bien des égards les tapis orientaux représentent donc un défi pour le système représentationnel occidental, ou pour le « regard occidental » comme le formule Belting, tout en ayant été intégrés très tôt dans l'espace pictural européen, comme nous l'avons vu. C'est cette tension entre étrangeté et familiarité que la narration historique essaie de résoudre à l'époque. Les recherches sur les tapis d'orient sont effectivement associées durant les dernières décennies du XIXe siècle à l'analyse de l'ornement de surface oriental (orientalische Flächenornamentik), conçu non seulement comme une technique mais comme un mode de perception et de représentation propre à certaines cultures. L'œuvre de l'historien de l'art viennois Alois Riegl constitue un moment essentiel de cette transition.

Alois Riegl, alors conservateur du département des textiles du Musée d'art et d'industrie de Vienne ${ }^{52}$, fut associé à l'exposition de tapis de 1891 en tant qu'assistant. Pour le catalogue, il rédigea une introduction sur l'histoire du tapis d'orient et une présentation de ses modes de fabrication ${ }^{53}$. Pour le livre publié à l'issue de l'exposition, il prépara les longs commentaires très détaillés et très précis des illustrations (Bildbeschreibungen ${ }^{54}$ ). Mais, surtout, c'est en rapport étroit avec la préparation ce cette exposition qu'il fait paraitre en 1891 son propre livre sur les anciens tapis orientaux, Altorientalische Teppiche. Lui aussi propose une classification des tapis d'après leur mode de fabrication (tapis noués, tapis tissés) et leur origine présumée, analysée en partie grâce aux techniques, aux 
couleurs et aux motifs. Mais contrairement à Lessing et en partie à Bode, il ne part plus des tableaux, mais des tapis eux-mêmes, atteint ainsi une plus grande précision dans l'analyse des techniques et des motifs, et, surtout, parvient à intégrer ceux-ci véritablement dans un récit englobant.

Selon Riegl, la tendance à développer un système de décoration couvrant la totalité des surfaces (Flächendekorationssystem) ne s'est pas imposée seulement avec l'apparition de l'Islam et l'extension de la domination arabe en Orient, mais prévalait déjà à une époque où l'antiquité tardive possédait encore une "validité universelle » (universale Geltung) ${ }^{55}$. Pour prouver cela, il fallait attester de manière détaillée une parenté interne entre les éléments décoratifs de l'antiquité tardive et les éléments sarrasins et, plus encore, il fallait suivre à la trace le processus par lequel les formes d'origine avaient acquis « suite à leur adaptation progressive au schéma d'une décoration de toute la surface, ce caractère transformé (...) qui dans l'art sarrasin nous fait face de manière si étrange et, en apparence, totalement étrangère $\aleph^{56}$. Le quatrième chapitre de l'ouvrage analyse les rapports entre les tapis noués et l'art oriental ancien en général, ainsi que les principales hypothèses concernant l'origine des tapis orientaux. Riegl s'y oppose de manière décidée à ce qu'il considère comme une "répartition anglaise " selon laquelle l'ornement en vrilles fleuries (Blütenrankenornament) serait une «langue formelle aryenne », tandis que l'ornement géométrique serait d'origine turkmène, c'est-à-dire mongole. Il n'existe pas selon lui de style originel commun aux peuples aryens (gemein-arischer Urstil) mais il exista bien «une période où ces pays et ces peuples se sont trouvés sous une seule et même domination artistique et, pour la plus grande partie, également politique : l'Empire romain. C'est dans l'antiquité tardive et non pas dans un âge aryen mythique des tribus que l'on peut trouver les points d'attache pour les vrilles de l'ornementation sarrasine ${ }^{57}$.

Tout l'ouvrage est porté par une interrogation sous-jacente sur l'origine et sur la nature même de l'orient qui restera d'ailleurs un trait caractéristique de l'oeuvre de Riegl. Les tapis offraient une possibilité de réfléchir à la naissance de l'orient, si l'on peut dire, ou en tout cas d'un style ornemental considéré comme proprement oriental, tout comme ils posaient aussi la question d'une culture transcendant la séparation entre orient et occident. Le cinquième chapitre de l'ouvrage s'intéresse aux orientalismes européens passés, puis à l'importation et à l'imitation actuelles de modèles orientaux en Europe, liées à la question des échanges commerciaux en tant qu'ils peuvent être fondés sur des inégalités de développement technique et industriel tout autant que sociales. Riegl indique que l'on sait maintenant de source sûre que, contrairement à ce qu'affirmait Lessing, il a bien existé une pratique autochtone du nouage en Europe, mais presque exclusivement dans le domaine domestique (notamment en Scandinavie). Pour ce qui est d'une "imitation consciente " des tapis orientaux, et particulièrement des ouvrages de luxe, il montre que dans tous les pays entrant en ligne de compte, c'est-à-dire en France, en Italie et en Espagne, la présence de tapis orientaux était liée à des rapports commerciaux étroits avec l'orient, donc à des importations et non au développement d'un art autochtone comparable à celui de l'orient. On pouvait selon lui déjà pressentir le moment où les conditions économiques qui avaient entraîné la disparition de l'artisanat domestique en Europe, s'étendraient également au Proche-Orient, scellant ainsi définitivement le destin de l'antique technique des tapis tissés.

Si l'histoire du tapis d'orient devient ainsi une histoire des rapports entre orient et occident, le tapis d'orient lui-même et son ornementation est envisagé comme une archive des mélanges féconds qui marquèrent l'Antiquité tardive. Riegl conclut en disant 
qu'il faut poursuivre le travail de collecte et d'inventaire, mais dans un but purement historique, et que faire des recherches sur l'art populaire des populations slaves de l'Empire signifie d'ailleurs faire quelque chose pour la reconnaissance de leurs cultures et, selon une mission propre à l'Autriche selon lui, assurer une mission « de veille aux portes de l'orient » (Hochwacht an der Pforte des Orients ${ }^{58}$ ). On comprend combien la collecte et l'étude des tapis s'inscrit ici dans un récit qui participe d'un « orientalisme de la frontière ${ }^{59}$.

Parti des tapis et de leur ornementation, Riegl sera amené dans ses Questions de style (1893), en partie à cause des discours racialisants de l'époque, à détacher l'histoire des formes ornementales de leur contexte et à revendiquer une histoire immanente de cellesci, au sens où le passage de l'une à l'autre est déterminé selon lui par des raisons formelles. Poursuivant son étude de l'Antiquité tardive, déjà évoquée dans Altorientalische Tepiche comme âge des mélanges féconds, dans son ouvrage L'industrie d'art romaine tardive , il inscrit toutefois l'ornementation dans une évolution plus générale des modes de perception, allant de l'haptique (vision de la surface sans profondeur) à l'optique (vision en profondeur $\left.{ }^{60}\right)$. Cette question du rapport entre surface et profondeur est posée également par un autre art extra-européen, l'art japonais, qui suscita un engouement important en Europe suite à la première grande manifestation du japonisme lors de l'exposition universelle de 1873 à Vienne. La notion de surface fait le lien entre décoration planimétrique et art japonais, mais, surtout, elle ouvre sur une conception moderne de la matérialité ${ }^{61}$. Il existe également un lien entre l'ornementation planimétrique et la photographie, considérée souvent - surtout à l'époque - comme un art de la surface.

Plus généralement les motifs des tapis d'orient posaient la question de l'intermédialité. Car, si les tapis incarnent de manière privilégiée une certaine transculturalité ${ }^{62}$, le « voyage » de leurs ornements d'un support à l'autre est tout aussi frappant : du jardin au tapis (puisque les tapis persans par exemple sont conçus comme des jardins), du tapis au tableau, du tableau au dessin, à la photographie, à l'intérieur. Les choses se compliquent encore si l'on tient compte $d u$ fait que le tapis, lié comme tout le textile à l'industrialisation, interrogeait d'une certaine façon les conceptions usuelles du geste technique. Défini par l'orientaliste autrichien Joseph Karabacek comme une " peinture à l'aiguille $\aleph^{63}$, composé selon certains témoignages de motifs mémorisés sous forme de mélodies, chantées par un maître aux ouvrières ${ }^{64}$, l'art des tapis d'Orient et son imitation posait également la question de la mise en carte et de la sérialisation du motif, comme révolution à la fois technique et médiale ${ }^{65}$.

Elles se compliquent également si l'on tient compte du fait qu'avec la représentation des intérieurs, les tapis d'orient vont voyager de nouveau vers les tableaux, où ils tendent ensuite à perturber la relation entre l'arrière plan et le premier plan, le fond et le sujet, et à s'autonomiser. C'est en ce sens que la planéité et l'abstraction de nombreux motifs ornementaux, ouvrant à la fois sur la matérialité et sur le rythme, s'inscrivent en tant que "paradigme du tapis » (Joseph Mashek ${ }^{66}$ ) dans un grand récit historique cherchant à retracer l'invention de la modernité picturale.

Comme l'ont montré les exemples développés ci-dessus, le tapis d'orient, topos par excellence de l'orient, "epitomé de l'intérêt occidental pour les objets étrangers " ${ }^{67}$, fragment d'ailleurs cristallisant des fantasmes d'authenticité, tendu entre appropriation et exotisation, engage plus largement l'histoire des relations commerciales, culturelles et politiques entre « orient » et « occident » ainsi que la construction des espaces concernés 
en tant qu'entités culturelles au moyen de diverses pratiques narratives. Mais le tapis devient aussi le lieu où se révèle une narrativité propre au motif lui-même. Si l'on suit Alfred Gell, qui consacre un chapitre de son livre Art and Agency à l'art décoratif, les motifs compliqués dont il est difficile de saisir le fondement mathématique ou géométrique seulement par l'inspection visuelle - il cite le cas du tapis d'orient-, engendrent des relations entre personnes et objets sur le long terme, car d'un point de vue cognitif ils sont toujours un « travail inachevé » (unfinished business) qui se poursuit dans des allers retours interminables ${ }^{68}$. Cela rejoint d'une certaine manière son analyse de «la technologie de l'enchantement» comme découlant d'un "enchantement de la technologie", au sens où toute virtuosité empêche l'observateur de comprendre la technique qui est à l'oeuvre ${ }^{69}$. Si le motif est donc un "travail inachevé », le tapis d'orient, et à travers lui la relation entre orient et occident, apparait elle-même comme un « unfinished business ».

Il est frappant effectivement de voir combien la capacité du tapis d'orient à conjuguer le familier et l'étranger a perduré et combien artistes ou conservateurs en jouent encore aujourd'hui. En 2012/2013 Philippe-Alain Michaud a organisé, d'abord à la Villa Médicis à Rome puis aux Abattoirs de Toulouse, une exposition intitulée «Tapis volants » jouant avec l'idée de lévitation mais centrée également sur un rapprochement complexe entre tapis et cinéma ${ }^{70}$. En 2013 Rudolf Stingel a moquetté le sol et les murs du Palazzo Grassi à Venise de reproductions agrandies et pixellisées d'un tapis azerbaïdjanais aux dominantes rouges et noires et y a éparpillé trente-quatre toiles, notamment - au dernier étage - des peintures photo-réalistes de statues de saints et de madones en noir, blancs et gris $^{71}$ rappelant, entre autres, les expérimentations de Bode avec une Madonne et un tapis persan évoquées précédemment. Enfin, en 2014, a été inaugurée la nouvelle installation de la célèbre collection de tapis d'orient du musée des arts décoratifs (MAK) de Vienne. Elle fut confiée à Michael Embacher, qui réalisa un ensemble censé traduire «les interconnexions géographiques, formelles, matérielles et techniques dans le développement du tapis noué entre le XVe et le XVIIIe siècle (...) dans le concept architectonique de la nouvelle installation »: les tapis ont été suspendus au moyen de câbles selon des angles différents qui permettent de subtils jeux de lumière, et donnent l'impression de «voler" tout en créant un espace semblable à un cocon de ver à soie ${ }^{72}$. Espace et narration se croisent ici de manière aussi intime que durant les dernières décennies du XIXe siècle, notamment dans des lieux stratégiques pour l'histoire du tapis d'orient, comme Venise ou Vienne.

\section{NOTES}

1. Brian Spooner, "Weavers and dealers: the authenticity of an oriental carpet ", dans : Arjun Appadurai (éd.), The Social life of things: commodities in cultural perspective, Cambridge University Press, 1986, p. 195-235.

2. Angela Völker, Die orientalischen Knüpfteppiche im MAK, Vienne, Böhlau, 2001 ; et de la même, «Die Sammlungspolitik der Textilsammlung des k.k. Österreichischen Museums für Kunst und 
Industrie in den Jahren 1864 bis 1910 », dans : Peter Noever (éd.), Kunst und Industrie. Die Anfänge des Museums für angewandte Kunst in Wien, Vienne, Hatje Cantz Verlag, 2000.

3. Angela Völker, Die orientalischen Knüpfteppiche im MAK, p. 10.

4. Voir par exemple cet ouvrage un peu plus tardif: Heinz Uhlemann, Geographie des Orientteppichs, Leipzig, K.W. Hiersemann, 1930.

5. Hans-Günther Schwarz, Orient - Okzident. Der orientalische Teppich in der westlichen Literatur, Ästhetik und Kunst, Munich, iudicium verlag, 1990.

6. Gottfried Semper, Der Stil in den technischen und tektonischen Künsten, oder praktische Ästhetik, vol. I : Francfort, Verlag für Kunst und Wissenschaft, 1860 ; vol. II : Munich, F. Bruckmann's Verlag, 1863.

7. Julius Lessing, Aufzeichnungen über Leben und Familie, tapuscrit s.d., Kunstgewerbe-Museum Berlin.

8. Julius Lessing, Altorientalische Teppichmuster nach Bildern und Originalen des XV.-XVI. Jahrhunderts, Berlin, Wasmuth, 1877.

9. Julius Lessing, Ancient Oriental Carpet Patterns after Pictures and Originals of the Fifteenth and Sixteenth Centuries, Londres, H. Sotheran \& Co., 1879; du même, Modèles de tapis orientaux, d'après des documents authentiques et les principaux tableaux du XVe et du XVIe siècles, Paris, Firmin-Didot, 1879.

10. Julius Lessing, Altorientalische Teppichmuster, p. 5.

11. Ibid., p. 5.

12. Ibid., p. 6.

13. Ibid., p. 6.

14. Ibid., p. 7.

15. Ibid., p. 7.

16. Ibid., p. 9.

17. Ibid., p. 12 .

18. Julius Lessing, Orientalische Teppiche, Vorbilder-Hefte aus dem Kgl. Kunstgewerbe-Museum, Heft 13, Berlin, Wasmuth, 1891.

19. Ibid., p. 2.

20. Johannes Wieninger, «Das Orientalische Museum in Wien, 1874-1906 », dans : Dieter Hornig, Johanna Borsch et Johannes Feichtinger (éds.), Vienne, porta Orientis, Austriaca 74/2012; Voir également Wolfgang Kos et Ralph Gleis (éds.), Experiment Metropole -1873: Wien und die Weltausstellung Ausstellungskatalog, Vienne, Wien Museum Czernin Verlag, 2014.

21. Johannes Wieninger. " "Er brachte viel eigenartiges und Notwendiges mit". Arthur von Scala als Mittler zwischen Ost und West und die Grundlegung der Asiensammlung des heutigen Museums für angewandte Kunst 1868 - 1909 » dans : Peter Noever (éd.), Kunst und Industrie. Die Anfänge des Museums für angewandte Kunst in Wien, Vienne, Hatje Cantz Verlag, 2000.

22. Programme de la journée d'étude "Collecting to Exhibit? The Narratives of Art Between Collection and Exhibition », 20 janvier 2016, ARoS Aarhus Art Museum, Danemark.

23. Voir Arthur von Scala, « Vorwort », dans : Katalog der Ausstellung orientalischer Teppiche im k.k. öterreichischen Handelsmuseum 1891, mit zahlreichen Holzschnitten, Wien, Verlag des k.k. österreichischen Handels-Museums, 1891, p. 5-10, ici p. 9.

24. « Aussteller-Verzeichnis », dans : Katalog der Ausstellung orientalischer Teppiche, p. I-II.

25. Voir Arthur von Scala, « Vorwort », dans : Katalog der Ausstellung orientalischer Teppiche, p. 7-8.

26. Wilhelm von Bode, Mein Leben, Berlin, 1930, Bd. 1, p. 124. Voir Volkmar Enderlein, Wilhelm von Bode und die Berliner Teppichsammlung, Berlin, Staatliche Museen $\mathrm{zu}$ Berlin-Preussischer Kulturbesitz, 1995, p. 15.

27. Wilhelm von Bode, Mein Leben, Berlin, 1930, Bd 2, p. 93. Voir Volkmar Enderlein, Wilhelm von Bode und die Berliner Teppichsammlung, p. 10. 
28. Wilhelm von Bode, «Ein altpersischer Teppich im Besitz der Königlichen Museen zu Berlin, Studien zur Geschichte der Westasiatischen Knüpfteppiche », dans : Jahrbuch der Kgl. Preussischen Kunstsammlungen, $\mathrm{n}^{\circ} 13$, Berlin, 1892, p. 26-49 et 108-137.

29. Wilhelm von Bode, «Altorientalische Thierteppiche ", dans : Orientalische Teppiche, 1892, p. VXII.

30. Wilhelm von Bode, Vorderasiatische Knüpfteppiche aus älterer Zeit, Leipzig, Hermann Seemann, 1901.

31. Wilhelm von Bode, Vorderasiatische Knüpfteppiche, p. 4. Voir Volkmar Enderlein, Wilhelm von Bode und die Berliner Teppichsammlung, p. 12-13.

32. Voir Volkmar Enderlein, Wilhelm von Bode und die Berliner Teppichsammlung, p. 17.

33. Peter Plener, "Sehnsüchte einer Weltausstellung-Wien 1873 », dans : kakanien revisted, 10 octobre 2001, p. 3 : http://www.kakanien-revisited.at/beitr/fallstudie/PPlener1.pdf

34. Dora Heinz, Linzer Teppiche. Zur Geschichte einer österreichischen Teppichfabrik der Biedermeierzeit, Vienne/Munich, Verlag Anton Schrol \& Co, 1955.

35. Voir Werner Telesko, Die Wiener Hofburg 1835-1918: Der Ausbau der Residenz vom Vormärz bis zum Ende des "Kaiserforums", Vienne, Verlag der österreichischen Akademie der Wissenschaften, 2012.

36. Eine Orientreise vom Jahre 1881, beschrieben vom Kronprinzen Rudolf von Österreich, illustrirt mit Holzschnitten nach Original-Zeichnungen von Franz von Pausinger, Vienne, Verlag der kaiserlichköniglichen Hof- und Staatsdruckerei, 1885.

37. Eva B. Ottilinger, « Kronprinz Rudolfs "Türkisches Zimmer" und die Orientmode in Wien », dans : Erika Mayr-Oehring (éd.), Orient : österreichische Malerei zwischen 1848 und 1914, catalogue de l'exposition du même nom à la Residenzgalerie Salzbourg (20.7. - 24.9.1997), Salzbourg, Residenzgalerie Salzburg, 1997, p. 99. Cette « chambre turque » est exposée aujourd'hui au musée du Hofmobiliendepot à Vienne.

38. M. P. M. Sekler, «Le Corbusier und das Museum als eine Stätte des Lernens », dans : Peter Noever (éd.), Kunst und Industrie. Die Anfänge des Museums für Angewandte Kunst in Wien, Vienne, MAK/Hatje Cantz Verlag, 2000, p. 252-270.

39. Voir notamment Jürgen Reulecke (éd.), Geschichte des Wohnens, vol. 3 : 1800-1918. Das bürgerliche Zeitalter, Stuttgart, Deutsche Verlagsanstalt, 1997.

40. Alois Riegl, Altorientalische Teppiche, Leipzig, T.O. Weigel, 1891, p. IV.

41. Carl Bötticher, Die Tektonik der Hellenen, Erstes Buch, Potsdam, 1852, p. 146.

42. Voir notamment Gottfried Semper, Die vier Elemente der Baukunst. Ein Beitrag zur vergleichenden Baukunde, Braunschweig, 1851.

43. Jacob von Falke, Die Kunstindustrie auf der Wiener Weltausstellung 1873, Vienne, Carl Gerold's Sohn, 1873, p. 166.

44. Ibid., p. 355-356.

45. Jakob von Falke, Die Kunst im Hause. Geschichtliche und kritisch-ästhetische Studien ueber die Decoration und Ausstattung der Wohnung, Vienne, C. Gerold's Sohn, 1871.

46. Jakob von Falke, Die Kunstindustrie auf der Wiener Weltausstellung 1873, p. 355.

47. Voir par exemple Jakob von Falke, Die Kunst im Hause, p. 259.

48. M. P. M. Sekler, « Le Corbusier und das Museum als eine Stätte des Lernens », p. 252-270.

49. Jean-Michel Rabaté, "L'image du tapis: de Holbein à Freud, en passant par James et par Joyce ", dans : Decorum. Tapis et tapisseries d'artiste, catalogue de l'exposition du même nom, Paris 11 octobre 2013-9 février 2014, Paris, 2014, p. 37-42.

50. Voir notamment: Erika Greber, Textile Texte: poetologische Metaphorik und Literaturtheorie. Studien zur Tradition des Wortflechtens und der Kombinatorik, Cologne/Weimar/Vienne, Böhlau, 2002 ; et Françoise Bort et Valérie Dupont (éds.), Texte, texture, textile : variations sur le tissage dans la musique, les arts plastiques et la littérature, Dijon, Editions universitaires de Dijon, 2013. 
51. Hans Belting, Florenz und Bagdad. Eine westöstliche Geschichte des Blicks, Munich, Beck, 2008 (édition poche 2012). Traduction française : Florence et Bagdad. Une histoire du regard entre Orient et Occident, trad. de l'allemand par Naïma Ghermani et Audrey Rieber, Paris, Gallimard, 2012.

52. Voir Céline Trautmann-Waller, «Etudier les tapis orientaux à Vienne en 1891: les débuts d'Alois Riegl », dans : Kerstin Hausbei et Alain Lattard (éds.), Identités multiples. Mélanges offerts à Gerald Stieg, Paris, 2008, p. 211-219.

53. Alois Riegl, "Geschichte des orientalischen Teppichs", dans: Katalog der Ausstellung orientalischer Teppiche, p. 11-23 ; du même, « Zur Herstellung der Knüpfteppiche », ibid., p. 24-27.

54. Alois Riegl, «Beschreibungen der auf den Tafeln I-CI abgebildeten Teppiche» dans: Orientalische Teppiche, Vienne, 1892, p. 1-22.

55. Alois Riegl, Altorientalische Teppiche, Vorwort, p. IX.

56. Ibid., p. X.

57. Ibid., p. 50.

58. Ibid., p. 76.

59. Andre Gingrich, "Kulturgeschichte, Wissenschaft und Orientalismus. Zur Diskussion des "frontier orientalism" in der Spätzeit der k.u.k. Monarchie ", dans: Johannes Feichtinger, Elisabeth Grossegger, Gertraud Marinelli-König, Peter Stachel et Heidemarie Uhl (éds.), Schauplatz Kultur - Zentraleuropa. Transdisziplinäre Annäherungen, Innsbruck, Vienne, Bozen, 2006, p. 279-288.

60. Alois Riegl, Die Spätrömische Kunstindustrie nach den Funden in Österreich-Ungarn dargestellt, Vienne, K. K. Hof- und Staatsdruckerei, 1901. Traduction française : L'industrie d'art romaine tardive, trad. par Marielène Weber, Sophie Yersin Legrand, Paris, Macula, 2014.

61. Voir notamment Sabeth Buchmann, Rike Frank (éds.), Textile Theorien der Moderne. Alois Riegl in der Kunstkritik, Berlin, b_books, 2015.

62. Voir à ce sujet, dans une perspective plus large : Annette Tietenberg (éd.), Muster im Transfer. Ein Modell transkultureller Verflechtung ?, Cologne, Böhlau, 2015.

63. Joseph Karabacek, Die persische Nadelmalerei Susandschird. Ein Beitrag zur Entwicklungs-Geschichte der Tapisserie de haute lisse. Mit Zugrundelegung eines aufgefundenen Wandteppichs nach morgenländischen Quellen dargestellt, Leipzig, Seemann, 1881.

64. Ahmad Seyf, «Carpet Manufactures of Iran in the Nineteenth Century », dans : Middle Eastern Studies, 26(2), avril 1990, p. 204-213, ici p. 206.

65. Birgit Schneider, Textiles Prozessieren. Eine Mediengeschichte der Lochkartenweberei, Berlin / Zurich, 2007.

66. Voir Joseph Mashek, Le Paradigme du tapis. Prolégomènes critiques à une théorie de la planéité, Genève, mamco, 2011 ; et du même, «Le paradigme du tapis » revisité, dans : Decorum. Tapis et tapisseries d'artiste, p. 95-102.

67. Brian Spooner, « Weavers and dealers », p. 195.

68. Alfred Gell, Art and Agency. An Anthropological Theory, Oxford, Clarendon Press, 1998, p. 80-81.

69. Alfred Gell, «The technology of enchantment and the enchantment of technology », dans J. Coote et A. Shelton (éds.), Anthropology, Art and Aesthetics, Oxford, Clarendon Press, 1992, p. 40-67.

70. Voir Philippe-Alain Michaud, Tapis volants, Catalogue de l'exposition du même nom, Rome, Drago, 2012.

71. Véronique Millet, «Compte rendu critique de l'expo Stingel - Rudolf Stingel s'empare du Palazzo Grassi ", in: Revue Ex_situ, publié en ligne le 26 septembre 2013: https:// revueexsitu.com/2013/09/26/compte-rendu-critique-de-lexpo-stingel-par-veronique-millet/ 72. «Wir wollen mit der Schönheit der Teppiche berühren. Gespräch zwischen Michael Embacher und Barbara Karl », dans : MAK/Guide Teppiche/Carpets, Vienne, 2014, p. 62-64, ici p. 62. 


\section{ABSTRACTS}

Cet article étudie comment ce qu'on pourrait appeler l'« ère bourgeoise » du tapis d'orient, met en relation, au moyen de divers types de récit, des espaces «orientaux » concrets et un orient mythique, l'espace du musée ou de l'intérieur et l'espace figuratif du tapis lui-même. Il s'appuie plus précisément sur les premières publications scientifiques sur les tapis d'orient, comme celles des historiens d'art allemands et autrichiens Julius Lessing $(1877,1891)$, Wilhelm Bode (1892, 1901), ou Alois Riegl (1891), le manuel de décoration de Jakob von Falke (1871), la première exposition consacrée exclusivement aux tapis d'orient à Vienne en 1891, et d'autres expérimentations muséographiques avec ce dernier. L'analyse vise à montrer comment la relation au tapis d'orient, tendue entre le familier et l'exotique, entre appropriation et défamiliarisation, constituait un « défi narratif » auquel on tenta de répondre au moyen de récits textuels ou au moyen de récits incarnés par des mises en scènes d'espaces comme le musée ou l'intérieur. Tandis que les voyages des motifs, de pays en pays, mais aussi de support en support, donnent lieu aux premières esquisses d'une histoire de l'art globale et hybride, mettant en rapport des espaces très éloignées et dépassant le clivage entre Orient et Occident, l'étude du système ornemental des tapis conduit à des travaux relatifs à une histoire culturelle de la perception et de la représentation de l'espace. Celle-ci s'intègre à son tour dans un " grand récit » qui est celui de l'émergence de la modernité picturale.

Dieser Beitrag untersucht, wie das, was man das «bügerliche Zeitalter des Orientteppichs » nennen könnte, anhand verschiedener Formen der Erzählung konkrete « orientale » Orte und den Orient als mythischen Ort, den Raum des Museums und des Interieurs und den figurativen Raum des Teppichs selbst miteinander in Beziehung setzt. Er stützt sich insbesondere auf die ersten wissenschaftlichen Untersuchungen zum Orientteppich, wie diejenigen der deutschen und österreichischen Kunsthistoriker Julius Lessing $(1877,1891)$, Wilhelm Bode $(1892,1901)$ oder Alois Riegl (1891), auf Jakob von Falkes Ratgeber Kunst im Haus (1871), auf die Wiener Ausstellung von 1891, die als erste ausschliesslich dem Orientteppich gewidmet war, und auf andere museographische Experimentierungen mit Orientteppichen. Die Analyse versucht zu zeigen, wie die Beziehung zum Orientteppich, die zwischen dem Vertrauten und dem Exotischen, der Aneignung und der Verfremdung gespannt war, eine "narrative Herausforderung » darstellte, die man versuchte durch textuelle Erzählungen oder durch Erzählungen, die durch räumliche Inszenierungen im Museum oder im Interieur verkörpert waren, zu beantworten, Während die Reisen der Muster von einem Land ins andere, aber auch von einem Medium ins andere, Anlass für erste Formen einer globalen und hybriden Kunstgeschichte sind, die jenseits der Trennung zwischen dem Orientalischen und dem Abendländischen weit von einander entfernte Räume miteinander in Beziehung setzt, führt die Untersuchung des ornamentalen Systems der Orientteppiche zu Forschungen über die Kulturgeschichte der Wahrnehmung und der Darstellung von Raum. Diese fügen sich ihrerseits in eine andere "grosse Erzählung », die der Entstehung der bildlichen Moderne. 


\section{INDEX}

Chronological index: 1875-1920, XIXe siècle, XXe siècle

Mots-clés: arts décoratifs, musées, intérieurs, art oriental, ornement

Geographical index: monde germanique, Orient, Turquie, Perse, Chine, Asie centrale

\section{AUTHOR}

\section{CÉLINE TRAUTMANN-WALLER}

Céline Trautmann-Waller est professeur en études germaniques à l'Université Sorbonne nouvelle-Paris 3. Elle dirige depuis 2008 le Centre d'études et de recherches sur l'espace germanophone (CEREG, Université Paris 3). Ses recherches portent sur l'histoire culturelle allemande, plus particulièrement l'histoire des sciences humaines dans l'espace germanophone de la fin du XVIIIe au début du XXe siècle. Parmi ses publications récentes : Ignác Goldziher : un autre orientalisme? (dir.), 2011 ; L'École viennoise d'histoire de l'art (dir.), 2011 ; Berlin 1913-Paris 1937 : l'esthétique et la science de l'art à l'âge des congrès (co-dir.), 2016. 